\title{
Bathymetric Estimation Using MeRIS Images in Coastal Sea waters
}

\author{
Audrey Minghelli-Roman, Laurent Polidori, Sandrine Mathieu-Blanc, Lionel Loubersac, and François Cauneau
}

\begin{abstract}
Bathymetric estimation using remote sensing images has previously been applied to high spatial resolution imagery such as CASI, Ikonos or SPOT but not on medium spatial resolution images (i.e. MeRIS). This choice can be justified when there is a need to map the bathymetry on large areas. In this letter we present the results of the bathymetry estimation over a large known area, the Gulf of Lion (France), expanding over $270 * 180 \mathrm{~km}$.
\end{abstract}

Index Terms - MeRIS, coastal zone, MERIS.

\section{INTRODUCTION}

B ATHYMETRIC information is particularly important in coastal areas which often exhibit a high population density, heavy maritime traffic and vulnerable natural ecosystems like mangrove, lagoons or coral reefs. In many regions sea depth changes because of erosion and sedimentation processes and bathymetry must often be updated. Bathymetric surveying of shallow sea water is often performed by conventional shipbased acoustic surveys. However, this technique requires heavy and expensive equipments [1] as well as time consuming data processing [2]. This has encouraged the development of remote sensing based bathymetric techniques. The airborne LIDAR technique, based on laser telemetry, is still very expensive and available on aerial platform only, so that it covers limited areas [3]. Radar imagery is very sensitive to sea surface roughness which is influenced by bottom topography in shallow areas [4]. This technique is cost effective and suitable for large areas, but it can only provide shape location and not quantitative depth. Bathymetry can also be based on images taken at different tide heights, resulting in different iso-depth lines that can be interpolated. However, this method is limited to the intertidal strip in areas with large tide amplitude [5].

Multispectral satellite imagery has been used to produce bathymetric maps by considering pixel reflectance as a depth indicator [6]. The sea surface reflectance just above the surface can be described as a function of the bottom signal, the

Manuscript received October 23, 2006 ; revised December 4, 2006.

A. Minghelli-Roman is with the Le2I, Université de Bourgogne, UMR-CNRS 5158, 21078 DIJON Cedex, France (email : audrey.roman@u-bourogne.fr).

L. Polidori is with IRD/Cesbio, 31401 Toulouse Cedex 9, France

S. Mathieu-Blanc is with Alcatel Alenia Space Industries, 06156 Cannes la Bocca Cedex, France

L. Loubersac is with IFREMER, DEL-DRV LER/LR, 34203 Sète Cedex, France

F. Cauneau is with Ecole des Mines de Paris, BP207, 06904 Sophia-Antipolis Cedex, France

Digital Object Identifier 10.1109/LGRS.2007.890548 deep water reflectance, the diffuse attenuation coefficient of light in the water column and depth. The system is inverted to yield depth estimation [7]. This technique has already been applied to high spatial resolution imagery such as CASI [8], SPOT [9] or Quickbird imagery [10]. In this letter, we apply the method to a MeRIS image. The MeRIS sensor, launched on board ENVISAT in 2002, was designed for sea colour observation, with a $300 \mathrm{~m}$ spatial resolution, 15 programmable spectral bands and a 3 day revisit period [11]. Before MeRIS, ocean colour monitoring was usually based on optical remote sensing with spatial resolutions around $1 \mathrm{~km}$ with sensors like NOAA-AVHRR, POLDER or SeaWiFS, which cover one to several thousands of kilometres in a single swath, allowing very short revisit periods.

As the bathymetry in the Gulf of Lion is well known, we chose this geographic area to test and validate the method.

In this letter, the main steps of the bathymetric estimation method are described and the different adaptations to MeRIS imagery are detailed (i.e. choice of spectral bands and product level, use of suspended matter product, etc.). The results are presented and validated using digitized isobaths from SHOM (Service Hydrographique et Océanographique de la Marine in France) maps as a reference. Finally, the potential and limitations of this bathymetric estimation approach are discussed.

\section{METHOD}

The original method [12] consists in measuring the water attenuation by estimating the deep water reflectance and the water depth with the assumption that the bottom reflectance attenuation is only due to the water column and therefore to the depth. The water attenuation is also assumed to be homogenous. The water attenuation model [13] is given by

$$
\rho_{s}(\lambda)=\left[\rho_{b}(\lambda)-\rho_{w}(\lambda)\right] e^{-2 k(\lambda) z}+\rho_{w}(\lambda)
$$

where

$\begin{array}{ll}\rho_{s} & \text { surface reflectance; } \\ \rho_{b} & \text { seabed reflectance; } \\ \rho_{w} & \text { deep water reflectance; } \\ k & \text { diffuse attenuation, } \\ z & \text { depth. }\end{array}$

In this equation, $\rho_{s}$ is known (value in the image), $\rho_{w}$ can be averaged under the ROI of deep water, $\rho_{b}$ does not need to be known, $k$ need to be estimated and $z$ is the principal unknown. Equation (1) is a approximate radiation transport equation, $\mathrm{k}$ is assumed to be independent of depth and the diffuse attenuation for the downwelling irradiance equals the diffuse attenuation for the upwelling radiance. 


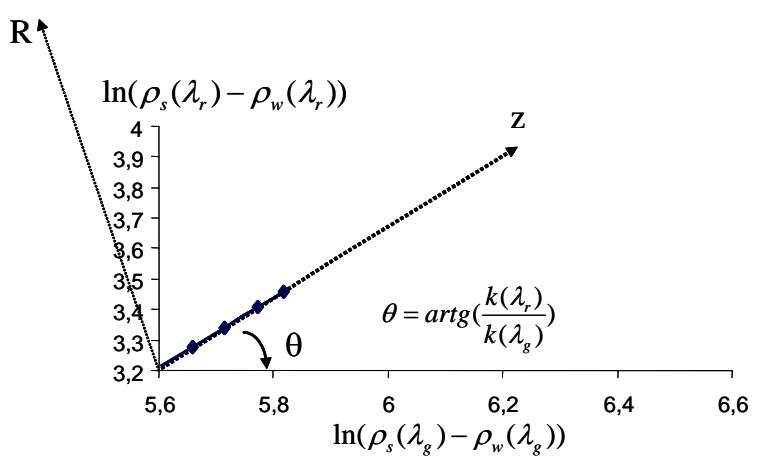

Fig. 1. Spectral rotation for bathymetric estimation [11].

The diffuse attenuation estimation requires different location sites (minimum 2) with the same type of cover, at different depths (not quantitatively known). For each site, pixels provide radiometric values for MeRIS wavelengths. The two useful wavelengths for this study are situated in the green $\left(\lambda_{\mathrm{g}}\right)$ and the red region $\left(\lambda_{\mathrm{r}}\right)$ as recommended by [12].

For the two spectral bands, we obtain two new bands $\mathrm{X}$ and $\mathrm{Y}$ from (1).

$$
\begin{aligned}
X & =\ln \left[\rho_{s}\left(\lambda_{g}\right)-\rho_{w}\left(\lambda_{g}\right)\right] \\
& =\ln \left[\rho_{b}\left(\lambda_{g}\right)-\rho_{w}\left(\lambda_{g}\right)\right]-2 k\left(\lambda_{g}\right) z \\
Y & =\ln \left[\rho_{s}\left(\lambda_{r}\right)-\rho_{w}\left(\lambda_{r}\right)\right] \\
& =\ln \left[\rho_{b}\left(\lambda_{r}\right)-\rho_{w}\left(\lambda_{r}\right)\right]-2 k\left(\lambda_{r}\right) z
\end{aligned}
$$

$\mathrm{Y}$ can be written as a linear expression of $\mathrm{X}$ whose slope equals the attenuation ratio of red and green regions $\left.\left.\left(\mathrm{k}\left(\lambda_{\mathrm{r}}\right)\right) / \mathrm{k}\left(\lambda_{\mathrm{g}}\right)\right)\right)$. This slope does not depend on the sea cover contrary to the $y$ intercept.

This theorical result is confirmed by the plot, on the same graph of different points corresponding to the different sites (different depths). We obtain effectively a line whose slope equals the attenuation ratio of red and green regions (Fig. 1).

The rotation of axes with an angle of $\left.\mathrm{k}\left(\lambda_{\mathrm{r}}\right)\right) / \mathrm{k}\left(\lambda_{\mathrm{g}}\right)$ ) ratio, provides two new images. This rotation aims at separating the influence of depth from other influences contributing to the measured reflectance. The depth-dependent image is the one along the axis marked "z". Several pixels corresponding to different depths are required to numerically evaluate the linear relation [12].

\section{Adaptation of the Method to MeRIS Images}

In the database of more than 50 images of the Golf of Lion, the selected image for the bathymetric estimation is the one having the higher visual correlation between radiometry and bathymetry (Fig. 2) but an other criterion is the suspended matter given by the MeRIS level 2 product. This choice is crucial for the estimation results because bathymetry result depend on the water transparency. For our study, the selected image has been acquired on August 22, 2003 and the suspended matter is lower than $1 \mathrm{~g} / \mathrm{m} 3$ within the image scene.

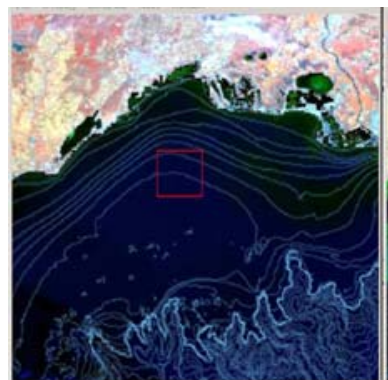

(a)

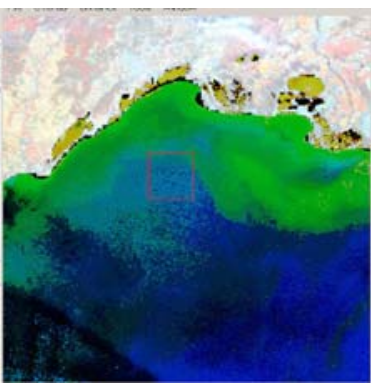

(b)
Fig. 2. (a) Isobaths on to the MeRIS image acquired on August, 22, 2003. (b) Radiometric equalization applied on the same image.

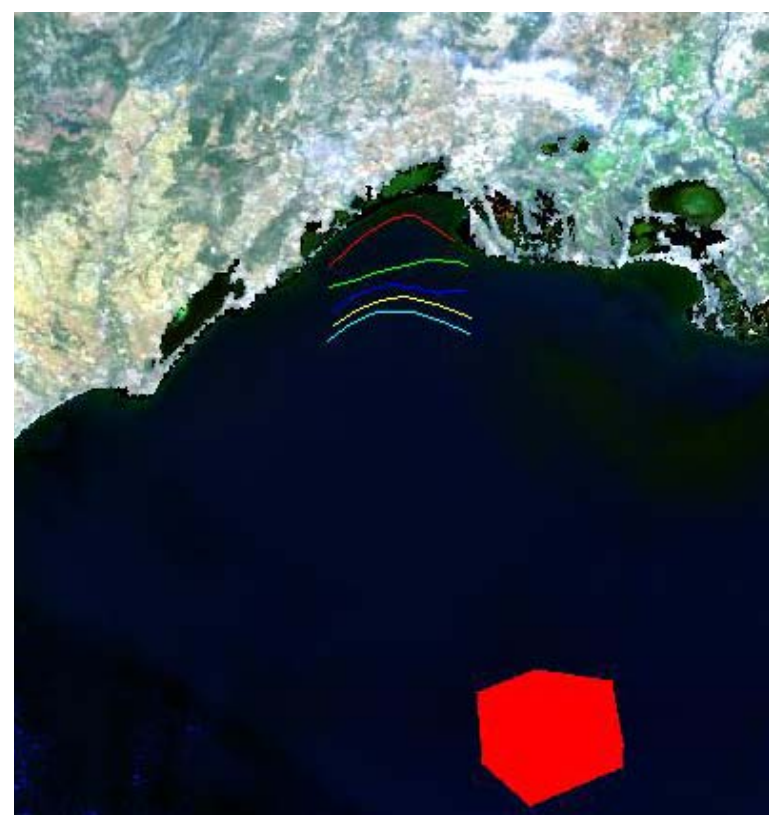

Fig. 3. ROI corresponding to 20-, 30-, 40-, 50-, 60-m isobaths, and deep water.

The validation data have been provided by digitized isobaths and used in a vector format. Some of the data are used as training values and the others are used for validation.

The MeRIS level 2 processing was chosen since the images are corrected for the atmospheric effects, which can change in different parts of the image.

The two MeRIS spectral bands are band 5 in the green region $(560 \mathrm{~nm})$ and band 10 in the red region $(750 \mathrm{~nm})$. This choice is empirical but the high-spectral resolution of MeRIS could help to accurately choose the optimal pair of bands.

The diffuse attenuation ratio $\mathrm{k}\left(\lambda_{\mathrm{r}}\right) / \mathrm{k}\left(\lambda_{\mathrm{g}}\right)$ has been estimated with different regions of interest (ROI), on different bathymetric lines at 20, 30, 40, 50 and $60 \mathrm{~m}$ (Fig. 3). The 10$\mathrm{m}$ line has not been used because MeRIS pixels at $10 \mathrm{~m}$ depth are close to the coastline and can even include it. 


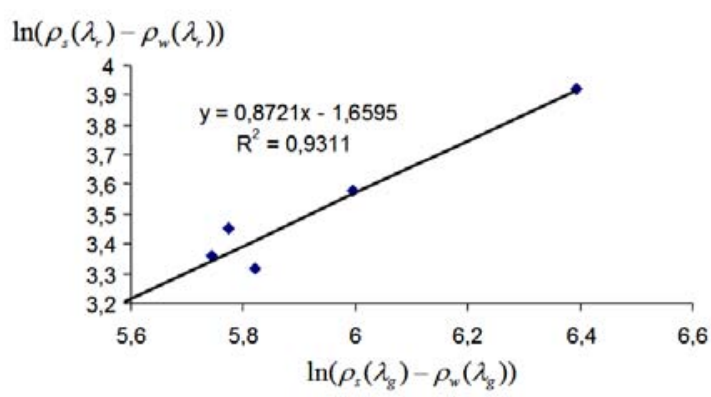

Fig. 4. Estimation of attenuation ratio $\mathrm{k}\left(\lambda_{\mathrm{r}}\right) / \mathrm{k}\left(\lambda_{\mathrm{g}}\right)$ with the mean radiometric values of each depth ROI.

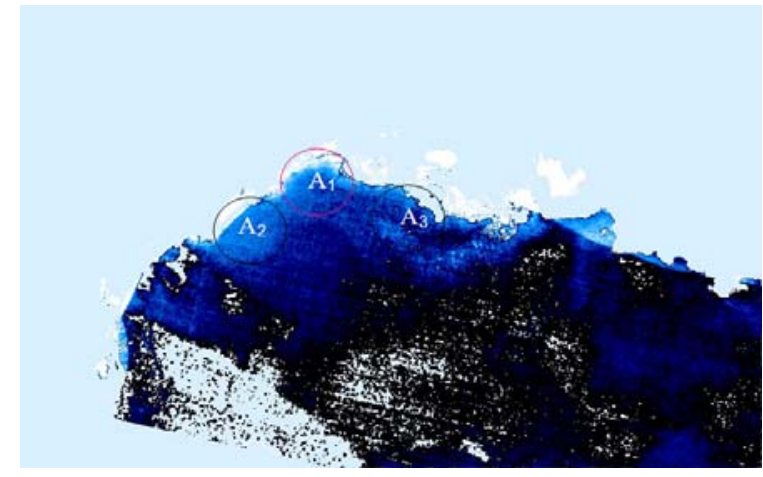

Fig. 5. Bathymetric image obtained. Colormap : white : shallow water ; dark blue : deep water.

If we plot the mean radiometric values of each ROI on the same graph, we obtain Fig. 4. These points are aligned with a correlation coefficient of 0.93 (Fig. 4).

Fig. 4 gives a ratio $k\left(\lambda_{r}\right) / k\left(\lambda_{g}\right)$ of 0,872 . Rotating the axes by an angle of $\operatorname{arctg}\left(k\left(\lambda_{\mathrm{r}}\right) / \mathrm{k}\left(\lambda_{\mathrm{g}}\right)\right)$ provides two new images. One of the resulting images is linearly dependant on depth. The calibration is given by fitting the reflectance of a few pixels with their known depth values. In the case of a linear calibration, the depth is obtained as

$$
Z=a\left[\ln \left[\rho_{s}\left(\lambda_{g}\right)-\rho_{w}\left(\lambda_{g}\right)\right]+\frac{k\left(\lambda_{r}\right)}{k\left(\lambda_{g}\right)} \ln \left[\rho_{s}\left(\lambda_{r}\right)-\rho_{w}\left(\lambda_{r}\right)\right]\right]+b
$$

The calibration constants $a$ and $b$ depend on the magnitude of the $\mathrm{k}$ values and the sea bed reflectance.

\section{RESUlts}

The resulting depth image is shown in Fig. 5.

The white pixels in deep water correspond to invalid depth estimations. For these pixels, the radiometric values were lower than in the deep water zone defined by the user. This may be due to a sea surface effect or to the spatial heterogeneity of water attenuation.
Distance to the coast

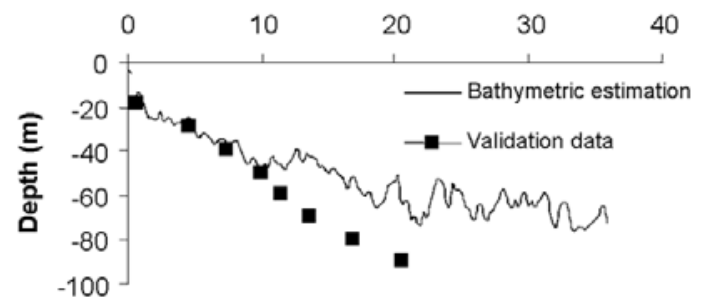

Fig. 6. Bathymetric Transect with validation points.

Three areas have been defined in the image.

1) The A1 area, used as a training area for the bathymetric model.

2) The $\mathrm{A} 2$ area, near the $\mathrm{A} 1$ area, used as validation site.

3) The A3 area, also used as a validation site. In this area, the water attenuation is not homogeneous because it is located near the Rhone estuary.

The isobaths obtained from digitalized maps have been used as ground truthing and the compared to model-derived values. These results are presented in tables I, II and III.

The last column of the tables gives the mean relative error.

A bathymetric transect has been plotted from the shore to the deep waters (Fig.6). For validation purposes, some real depths have been plotted on the graph. The comparison shows that the bathymetric estimation is rather accurate between 20 $\mathrm{m}$ and $50 \mathrm{~m}$ and becomes saturated and noisy when the depth is over $60 \mathrm{~m}$. This effect is due to the increasing absorption of the water column when the depth increases and therefore reducing the influence of seafloor signal.

\section{DisCUSSION}

These tables show that the bathymetric estimation is quite good when performed close to the training area. This is due to the low variation of the attenuation in a limited geographic area, as A1 and A2 areas. The mean relative errors are respectively 16 and $23 \%$ in these 2 areas.

For A3 area, located close to the Rhone estuary and close to the training area, the water attenuation is not only different from the case of the A1 area, but it also varies inside the area, resulting in a high mean relative error up to $52 \%$. This is a limitation of the homogeneity hypothesis, which is partly inherent to wide field-of-View (FOV) sensors like MeRIS, results in covering large water areas so that water attenuation may have significant spatial variations. In the A1 area, i.e. the training area, we could wish to have a null mean relative error. This is not the case since points in the plot (Fig. 4) are not perfectly aligned. This can be due to a slight difference of seabed cover for the points used for the model setup. Indeed the nature of sediments can vary with depth and therefore reduce the quality of the bathymetric estimation.

The medium resolution of $300 \mathrm{~m}$ increases the error of estimation for two reasons. First, in the coastal area, where the depth is less than $10 \mathrm{~m}$, the estimation is not accurate because each MeRIS pixel $(300 \mathrm{~m} * 300 \mathrm{~m})$ may contain a mixture of water, sand and rocks, contributing to estimation errors. Secondly, the depth estimation error increases with the seabed 
TABLE I

RESULT AND VALIDATION ON A1 AREA

\begin{tabular}{|c|c|c|c|c|}
\hline Mean depth $(\mathrm{m})$ & Estimated depth $(\mathrm{m})$ & Absolute error $(\mathrm{m})$ & Relative error & RMSE \\
\hline 20 & 16,1 & $-3,9$ & $-20 \%$ & 9,36 \\
\hline 30 & 36,6 & 6,6 & $22 \%$ & 12,05 \\
\hline 40 & 47,2 & 7,2 & $18 \%$ & 9,67 \\
\hline 50 & 47,4 & $-2,6$ & $-5 \%$ & 6,4 \\
\hline 60 & 51,7 & $-8,3$ & $-14 \%$ & 10,41 \\
\hline
\end{tabular}

TABLE II

RESUlT AND VALIDATION ON A2 AREA

\begin{tabular}{|c|c|c|c|c|}
\hline Mean depth (m) & Estimated depth (m) & Absolute error $(\mathrm{m})$ & Relative error & RMSE \\
\hline 20 & 28,0 & 8,0 & $40 \%$ & 9,52 \\
\hline 30 & 33,4 & 3,4 & $11 \%$ & 4,78 \\
\hline 40 & 36,7 & $-3,3$ & $-8 \%$ & 4,91 \\
\hline 50 & 39,0 & $-11,0$ & $-22 \%$ & 11,67 \\
\hline 60 & 40,8 & $-19,2$ & $-32 \%$ & 20,36 \\
\hline & & & $23 \%$ & 12,02 \\
\hline
\end{tabular}

TABLE III

RESULT AND VALIDATION ON A3 AREA

\begin{tabular}{|c|c|c|c|c|}
\hline Mean depth (m) & Estimated depth $(\mathrm{m})$ & Absolute error $(\mathrm{m})$ & Relative error & RMSE \\
\hline 20 & 39,7 & 19,7 & $99 \%$ & 21,59 \\
\hline 30 & 43,9 & 13,9 & $46 \%$ & 24,96 \\
\hline 40 & 61,1 & 21,1 & $53 \%$ & 25,15 \\
\hline 50 & 74,5 & 24,5 & $49 \%$ & 30,56 \\
\hline 60 & 68,1 & 8,1 & $14 \%$ & 18,54 \\
\hline
\end{tabular}

slope because each pixel covering $300 * 300 \mathrm{~m}$ provides the mean depth over this surface.

No technique is very accurate in terms of absolute depth estimation from medium spatial and spectral resolution sensors [14] except for inter-tidal area. However, a coarse bathymetry can provide a qualitative surface description and help marine specialists by revealing seabed morphology.

At this stage of the study, the spectral potential of MeRIS is not completely exploited because only 2 bands are used. A possible improvement could be to use a combination of several bands to optimise the results. Furthermore, this study site has been chosen because its bathymetry is well known and because there is no significant tide variation in this region. In a near future, we intend to apply this method on a less known area like the large and shallow New-Caledonian lagoon.

\section{CONCLUSION}

This letter shows that MeRIS imagery can be used for bathymetric estimation in shallow areas, excepted very close to the coast. This method can be used on a large area with clear and homogeneous water and it requires at minimum two known depths to train the model. The range of coherent estimation has been determined between $20 \mathrm{~m}$ and $50 \mathrm{~m}$ when the water attenuation is weak with an estimation error of $16 \%$ (table 1). This error increases with the distance to the training area when the water attenuation is not homogenous. This study confirms the potential of MeRIS for coastal water monitoring.

\section{REFERENCES}

[1] E. Kammerer, D. Charlot, S. Guillaudeux and P. Michaux, "Comparative study of shallow water multibeam imagery for cleaningbathymetry sounding errors”, OCEANS, 2001. MTS/IEEE Conference and Exhibition, vol.4, pp. 2124-2128, 2001

[2] J.S. Bird, G.K. Mullins, "Analysis of swath bathymetry sonar accuracy", IEEE J. Oceanic Engineering, vol.30, pp. 372-390, 2005

[3] H. Wong, A. Antoniou, "One-dimensional signal processing techniques for airborne laser bathyemetry”, IEEE Trans. Geosc. Remote Sensing, vol. 32, pp. 35-46, 1994

[4] W. Alpers, I. Hennings, "A theory of the imaging mechanism of underwater bottom topography by real and synthetic aperture radar", Journal of Geophysical Research 89, 10529-10546, 1984.

[5] N. Gratiot, A. Gardel, L. Polidori, "Remote sensing Based Bathymetry on highly dynamic Amazonian coast, 9th International Coastal Symposium, Iceland, 6-8 June, 2005.

[6] F.C. Polcyn, W.L. Brown, I.J. Sattinger, "The measurement of water depth by remote sensing”, Report no. 8973-26-F. Ann Arbor, MI, USA: Willow Run Laboratories. Michigan University, 1970

[7] D. Lyzenga, "Shallow-water bathymetry using combined lidar and passive multispectrale scanner data”, Int. J. Remote Sensing, vol. 6, pp. 115-125, 1985

[8] M. Despinoy, A. Minghelli-Roman, A. Begué, M. Petit, J. Coudray, A. Barcelo, "Airborne CASI imagery for bathymetric study in Reunion Island (Indian ocean)", IGARSS'03, pp. 2368-2370, 2003

[9] V. Lafon, J.M. Froidefond, F. Lahet and P. Castaing, "SPOT shallow water bathymetry of a moderately turbid tidal inlet based on field measurements", Remote Sensing Env., vol. 81, pp. 136-148, 2002

[10] S.M. Adler-Golden, P.K. Achaya, A. Berk, M.W. Mattew and D. Gorodetzky, "Remote bathymetry of the littoral zone from AVIRIS, LASH and Quickbird", IEEE Trans. Geosc. Remote Sensing, vol. 43, pp. 337-347, 2005

[11] M. Rast, J.L. Bézy and S. Bruzzi, "The ESA Medium Resolution Imaging Spectrometer MeRIS-a review of the instrument and its mission”, Int. J. Remote Sensing, vol. 20, pp. 1681-1702, 1999

[12] W. D. Philpot, "Bathymetric mapping with passive multispectral imagery", Applied optics, vol. 28, pp. 1569-1578, 1989

[13] S. Maritorena, A. Morel and B. Gentili, "Diffuse reflectance of oceanic shallow waters: influence of water depth and bottom albedo", Limnol. Oceanogr., vol. 39, 7, pp. 1689-1703, 1994

[14] S. Andréfouët, "Apport de la télédétection à une approche descriptive et fonctionnelle des systèmes coralliens de Polynésie Française”, PhD. Thesis, University of French Polynesia, 1998. 